\title{
UNIVERSITYOF
}

FORWARD

THINKING

WESTMINSTER用

WestminsterResearch

http://www.westminster.ac.uk/westminsterresearch

Multi-Party Trust Computation in Decentralized Environments

Dimitriou, T. and Michalas, A.

This is a copy of the author's accepted version of a paper subsequently published in the proceedings of the International Conference on New Technologies, Mobility and Security (NTMS), Istanbul 7-10 May 2012.

It is available online at:

https://dx.doi.org/10.1109/NTMS.2012.6208686

(C) 2012 IEEE . Personal use of this material is permitted. Permission from IEEE must be obtained for all other uses, in any current or future media, including reprinting/republishing this material for advertising or promotional purposes, creating new collective works, for resale or redistribution to servers or lists, or reuse of any copyrighted component of this work in other works.

The WestminsterResearch online digital archive at the University of Westminster aims to make the research output of the University available to a wider audience. Copyright and Moral Rights remain with the authors and/or copyright owners.

Whilst further distribution of specific materials from within this archive is forbidden, you may freely distribute the URL of WestminsterResearch: ((http://westminsterresearch.wmin.ac.uk/)).

In case of abuse or copyright appearing without permission e-mail repository@westminster.ac.uk 


\section{Multi-Party Trust Computation in Decentralized Environments}

\author{
Tassos Dimitriou \\ Athens Information Technology \\ 19.5 km Markopoulo Ave., 19002, Peania \\ Athens, Greece \\ Email: tdim@ait.edu.gr
}

\author{
Antonis Michalas \\ Athens Information Technology, Greece \\ and \\ Aalborg University, Denmark \\ Email: amic@ait.edu.gr
}

\begin{abstract}
In this paper, we describe a decentralized privacypreserving protocol for securely casting trust ratings in distributed reputation systems. Our protocol allows $n$ participants to cast their votes in a way that preserves the privacy of individual values against both internal and external attacks. The protocol is coupled with an extensive theoretical analysis in which we formally prove that our protocol is resistant to collusion against as many as $n-1$ corrupted nodes in the semi-honest model.

The behavior of our protocol is tested in a real P2P network by measuring its communication delay and processing overhead. The experimental results uncover the advantages of our protocol over previous works in the area; without sacrificing security, our decentralized protocol is shown to be almost one order of magnitude faster than the previous best protocol for providing anonymous feedback.
\end{abstract}

Index Terms-Decentralized Reputation Systems, Security, Voter Privacy, Anonymous feedback

\section{INTRODUCTION}

During the last decade, within the field of greatly developed online communities, unknown users may exchange information while keeping their identity hidden. However, the difficulty of gathering (reliable) evidence about unidentified transaction partners makes it hard to decide if a user is legitimate or corrupted. It is also difficult to differentiate between a high and a low quality service provider. As a result, the topic of trust is receiving significant attention in both the academic community and the e-commerce industry [7].

A well known technique used to prevent interaction with malicious or unreliable users is Reputation systems, which rates the behavior of each user, based on the quality of the provided service(s), and gives information to the community in order to decide whether to trust an entity in the network.

However, a relatively unexplored aspect of reputation systems is that of feedback providers' privacy. Only some reputation and trust establishment schemes deal with the problem of securing the ratings (or votes) of participating nodes. This lack of privacy can lead to several problems, including the proper operation of the network. Additionally, the absence of schemes that provide privacy in decentralized environments, such as ad hoc networks, is even greater. For example, it has been observed in [10] that users of a reputation system may avoid providing honest feedback in fear of retaliation, if reputation scores cannot be computed in a privacy-preserving manner. In response to that, eBay has decided to change the feedback policy so that sellers can no longer leave negative/neutral feedback for buyers, claiming that "it will help buyers leave an honest feedback" [5]. Hence, developing anonymous feedback reputation protocols is essential to online communities and electronic marketplaces, especially in decentralized environments that offer new challenges and opportunities for research.

Contribution: In this work we present a protocol that preserves the privacy of votes in decentralized environments. The protocol allows $n$ participants to securely cast their ratings in a way that preserves the privacy of individual votes against both internal and external attacks. More precisely, we analyze the protocol and prove that it is resistant to collusion even against up to $n-1$ corrupted insiders. The insights we obtain from this analysis allow us to refine the protocol and come up with a lighter version that is equally secure and uses only standard cryptographic mechanisms. This lighter protocol compares favorably with protocols for secure multi-party sum computation and we consider it as another important contribution of this work. Finally, the whole analysis is coupled with extensive experimental results that demonstrate the protocol's validity and efficiency over previous works in the area.

Organization of the paper: In Section II, we review some of the most important schemes that provide private trust ratings in decentralized environments. In Section III, we describe the problem of secure trust aggregation and we define the basic terms that we use in the rest of the paper. In Section IV, we present StR, our main protocol, while in Section V we provide a security discussion in which we show the resistance of our protocol against numerous attacks. Section VI describes the more efficient version of StR. In Section VII, we present experimental evidence that shows the effectiveness of our protocol, and, finally, Section VIII concludes this paper.

\section{RELATED WORK}

A limited number of reputation and trust establishment schemes deal with the problem of securing the vote(s) of each individual node. The difficulties of building reputation systems that can also preserve privacy can be found in [3]. Three works that work on the problem of computing ratings in decentralized reputation systems are those of [9], [6], [4].

Pavlov et al. [9] showed that when $n-1$ malicious nodes collude with the querying node to reveal the vote of the re- 
maining node then perfect privacy is not feasible. Furthermore, they proposed three protocols that allow voting to be privately provided in decentralized additive reputation systems. The first protocol is not resilient against collusion of nodes and can be used when dishonest peers are not an issue. The other two protocols are based on a probabilistic peers' selection scheme and are resistant to collusion of up to $n-1$ peers only with a certain degree of probability. Hasan et al. [6] proposed a privacy preserving protocol under the semi-honest adversarial model. It's main difference from Pavlov's protocols is that each $U_{i}$ sends her shares to at most $k<n-1$ nodes that are considered "trustworthy" by $U_{i}$.

Dolev et al. [4] proposed two main decentralized schemes where the number of messages exchanged is proportional to the number $n$ of participants (however, the length of each message is $O(n)$ ). The first protocol AP (and its weighted variant WAP) assumes that the querying agent $A_{q}$ is not compromised while the next protocol, namely MPKP (and its weighted variant MPWP) assumes that any node can act maliciously. The weakness of Dolev's protocols is the fact that unnecessary and inefficient computations are taking place.

One cannot help but notice the relevance of this problem to secure multi-party computation, where a number of distributed entities collaborate to compute a common function of their inputs while preserving the privacy of these inputs. One such well known example is the secure sum protocol [2], which uses randomization to securely compute the sum of the individual inputs. This protocol is a natural fit for the problem at hand but it suffers from a number of attacks and falls prey to honestbut-curious insiders which can easily infer the private input of any entity.

The protocols in [9], [6], [4] can be thought as attempts to recover from the security inefficiencies of secure sum, properly applied to the context of reputation management. Our final protocol, shown in Section VI, not only improves upon these schemes but can also be applied directly for secure sum computation, refining earlier results in this area [11].

\section{Problem Statement \& Definitions}

We start by providing a definition of decentralized additive reputation systems as described in [9].

Definition 1: A Reputation System $R$ is said to be a Decentralized Additive Reputation System if it satisfies the following two requirements:

1) Feedback collection, combination and propagation are implemented in a decentralized way.

2) Combination of feedbacks provided by nodes is calculated in an additive manner.

In this paper, we focus on the following problem:

Problem Statement: A querying node $A_{q}$, receives a service request from a target node $A_{t}$. Since $A_{q}$ has incomplete information about $A_{t}$, she asks other nodes in the network to give their votes about $A_{t}$. Let $U=\left\{U_{1}, \cdots, U_{n}\right\}$ be the set of all nodes that will provide an opinion to $A_{q}$. The problem is to find a way that each vote $\left(v_{i}\right)$ remains private while at the same time $A_{q}$ would be in position of understanding what voters, as a whole, believe about $A_{t}$, by evaluating the sum of all votes $\left(\sum_{i=1}^{n} v_{i}\right)$.

Similar to existing work in the area, all the protocols that are presented in this paper assume that the adversary is semihonest. In the semi-honest adversarial model, malicious nodes correctly follow the protocol specification. However, nodes overhear all messages and may attempt to use them in order to learn information that otherwise should remain private. Semihonest adversaries are also called honest-but-curious.

For the needs of our protocol, we assume that the reader is familiar with the concept of public key cryptography. Each node $\left(A_{q}, U_{i}, i \in[1, n]\right)$ has generated a public/private key pair $\left(k_{A_{q}} / K_{A_{q}}, k_{U_{i}} / K_{U_{i}}\right)$. The private key is kept secret, while the public key is shared with the rest of the nodes. These keys will be used to secure message exchanges between the nodes, hence the communication lines between parties are assumed to be secure. Our first protocol also relies on the use of homomorphic encryption for the collection of votes by the querying agent $A_{q}$. The vote of $U_{i}$ concerning $A_{t}$ is denoted by $v_{i}$. The notation $E($.$) will refer to the results of the application$ of an homomorphic encryption function that $A_{q}$ can decrypt with her private key. Pailler's Cryptosystem [8] is an example of cryptosystem where the trapdoor mechanism is based on such a homomorphic function.

\section{Splitting the Random Values (StR)}

In this section, we present our main protocol (StR) in which we use both homomorphic encryption and random numbers to secure the privacy of votes for each node.

During the initialization step, $A_{q}$ creates the set $U$ with all voters, orders them in a circle $A_{q} \rightarrow U_{1} \rightarrow \cdots \rightarrow U_{n}$ and sends to each $U_{i}$ the identity of its successor in the circle. Each $U_{i}$ splits its random number $r_{i}$ into $n$ pieces and shares one with the rest of the nodes. Then, it creates a blinded vote and adds it to the sum of previous votes by using the homomorphic property of Paillier's cryptosystem [8]. At the end, the last node $U_{n}$ forwards to $A_{q}$ the sum of all $n$ votes encrypted with the public key of $A_{q}$. Upon reception, $A_{q}$ decrypts the result and finds the sum of all votes. A detailed description of StR follows below.

First round: During the initialization step, $A_{q}$ sends to all nodes the list of all voters $U$. Each node $U_{i}$ generates a random number $r_{i}$ and splits it into $n$ integers in such a way that the $i^{\text {th }}$ share will be encrypted with the public key of $U_{i}$. So, if $U_{1}$ has generated a random number $r_{1}$, the shares will be

$$
r_{1}=r_{1,1},\left\{r_{1,2}\right\}_{k_{U_{2}}}, \cdots,\left\{r_{1, n-1}\right\}_{k_{U_{n-1}}},\left\{r_{1, n}\right\}_{k_{U_{n}}} .
$$

The next step for each $U_{i}$ is to distribute the shares to the remaining $n-1$ nodes in $U$. This means that each $U_{i}$ will receive the following $n-1$ messages

$$
\left\{r_{1, i}\right\}_{k_{U_{i}}}, \cdots,\left\{r_{i-1, i}\right\}_{k_{U_{i}}}, \cdots,\left\{r_{n-1, i}\right\}_{k_{U_{i}}},\left\{r_{n, i}\right\}_{k_{U_{i}}} .
$$

Since all $n-1$ numbers that $U_{i}$ received are encrypted with her public key, she decrypts them and calculates the blinded vote $b_{i}$ which is equal to 


$$
b_{i}=v_{i}+r_{i}-\left(\sum_{j=1}^{n} r_{j, i}\right) .
$$

When all nodes (in parallel) compute their blinded votes, the second round begins.

Second round: $U_{1}$ calculates $E\left(b_{1}\right)$ and sends it to $U_{2} . U_{2}$ adds $b_{2}$ to $E\left(b_{1}\right)$ by using the additive homomorphic property $\left(E\left(b_{1}\right) \cdot E\left(b_{2}\right)=E\left(b_{1}+b_{2}\right)\right)$ of Paillier's cryptosystem and sends $E\left(b_{1}+b_{2}\right)$ to $U_{3}$. At the end of this round $A_{q}$ will receive from $U_{n}$ the following: $E\left(\sum_{i=1}^{n} b_{i}\right)=E\left(\sum_{i=1}^{n} v_{i}\right)$. Upon reception, $A_{q}$ decrypts the message, finds the sum of all votes and divides by $n$ in order to find the average of votes. A concise description of StR is shown in Algorithm 1.

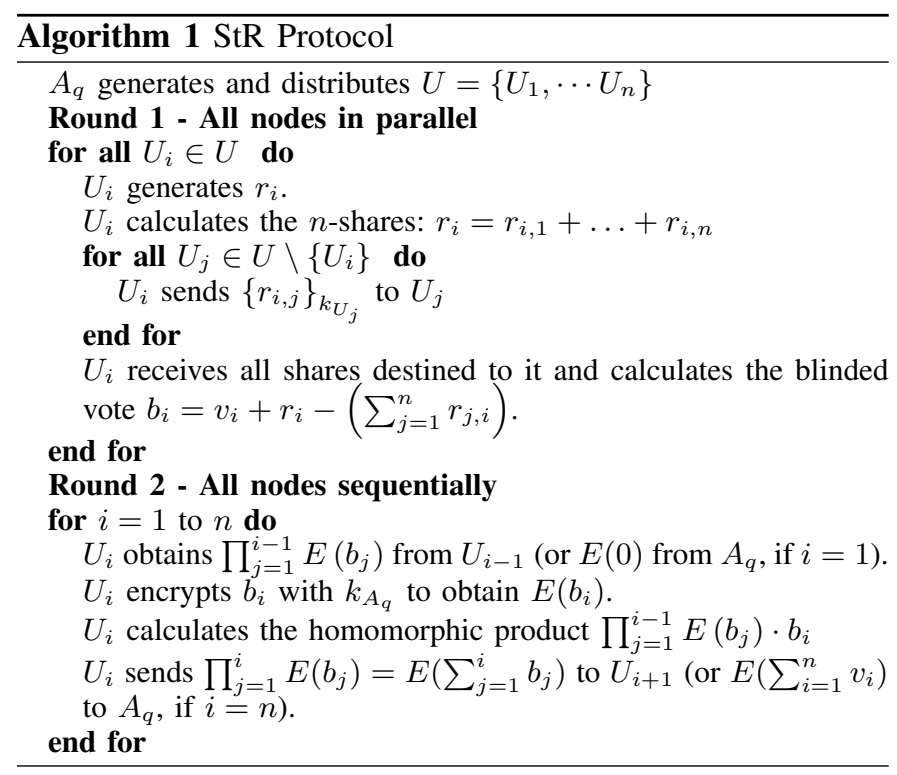

\section{SECURITy ANALYsis}

In this section we analyze the behavior of StR in the presence of corrupted agents. First, we will consider the case of a well-behaving query agent $A_{q}$. Such an agent respects the privacy of participating users and does not form malicious coalitions with corrupted agents in the set $U$ (however, among the agents in $U$ there can be corrupted ones). Then, in Section VI, we will proceed to discuss the case where $A_{q}$ is malicious as well. This will also lead to the development of an even more efficient but equally secure version of StR.

Theorem 1 (Uncompromised $A_{q}$ ): Assume an honest-butcurious adversary $\mathcal{A D V}$ corrupts at most $k<n$ users out of those in the set $U$. Then $\mathcal{A D} \mathcal{V}$ cannot infer any information about the votes of the legitimate users.

Proof. We will prove the privacy of the StR protocol using a standard simulation argument. In particular, we will show that for any adversary that corrupts (or controls) a subset of the participating users, there exists a simulator that, given the corrupted parties data and the final result, can generate a view that, to the adversary, it is indistinguishable from a real execution of the protocol. This guarantees that whatever information the adversary can obtain after attacking the protocol can be actually generated by herself, using the simulator. As a result, no useful information about legitimate users' data is leaked.

Let $C=\left\{U_{i_{1}}, U_{i_{2}}, \ldots, U_{i_{k}}\right\}$ denote the set of compromised users, where $k<n$. Let also view ${ }^{C}$ denote the views of the protocol for all users in $C$, including their votes $\left\{v_{i_{1}}, v_{i_{2}}\right.$, $\left.\ldots, v_{i_{k}}\right\}$, their random numbers $\left\{r_{i_{1}}, r_{i_{2}}, \ldots, r_{i_{k}}\right\}$ and the sequence of messages $E\left(\sum_{j=1}^{i_{1}} b_{j}\right), \ldots, E\left(\sum_{j=1}^{i_{k}} b_{j}\right)$ received by each one of them during the second round of the protocol, where by definition $b_{i}=v_{i}+r_{i}-\left(\sum_{j=1}^{n} r_{j, i}\right)$.

A simulator has access to the shares of the random numbers $r_{i, j}, i \neq j$ that ended up in corrupted users during the first round but cannot possibly generate the exact sequence of encrypted sums since it does not know the private data of legitimate users. So, the simulator will have to replace the private data with random quantities $\alpha_{i}$ and compute $E\left(b_{i}^{\prime}\right)$ for all $i=1, \ldots, n$, where $b_{i}^{\prime}=b_{i}$, if $U_{i}$ is corrupted/compromised and $b_{i}^{\prime}=\alpha_{i}$, otherwise. The simulator can now replace $E\left(\sum_{j=1}^{i_{l}} b_{j}\right)$ with $E\left(\sum_{j=1}^{i_{l}} b_{j}^{\prime}\right)$.

To complete the analysis we need to argue that if there exists an adversary $\mathcal{A}$ that distinguishes between the encryption of the observed values $E\left(\sum_{j=1}^{i_{l}} b_{j}\right)$ and the random ones $E\left(\sum_{j=1}^{i_{l}} b_{j}^{\prime}\right)$ produced by the simulator, then there is an adversary $\mathcal{B}$ that can attack the semantic security of $E(\cdot)$.

Such an attacker $\mathcal{B}$ would operate as follows: Its input is a sequence of values $E\left(x_{i}\right), i=1, \ldots, n$ and its goal is to determine whether the values $x_{i}$ correspond to the values provided by the users, or is simply a sequence of random values $\alpha_{i}$. Adversary $\mathcal{B}$, using the homomorphic property of $E()$, computes $E\left(\sum_{j=1}^{i_{l}} x_{j}\right)$ and provides the encryption of the partial sums $E\left(\sum_{j=1}^{i_{1}} x_{j}\right), \ldots, E\left(\sum_{j=1}^{i_{k}} x_{j}\right)$ as input to $\mathcal{A}$. It then returns whatever answer $\mathcal{A}$ returns.

Obviously $\mathcal{B}$ would be able to break the semantic security of $E()$ with the same probability that $\mathcal{A}$ could distinguish between the real views and the random values produced by the simulator. Since $E()$ is assumed to be semantically secure, such $\mathcal{A}$ cannot exist. Hence the security of the StR protocol is guaranteed provided at most $k<n$ users are compromised, but $A_{q}$ is not.

\section{A More EfFicient STR}

In this section we will consider the case where node $A_{q}$ is compromised as well. Since $A_{q}$ knows the private key and $A_{q}$ has been compromised by $\mathcal{A D V}$ (or is member of the colluding group), $A_{q}$ can simply decrypt any communicated message. Hence we cannot rely on the semantic security property of the underlying cryptosystem. In this scenario the security is therefore solely based on the randomness which is used to blind the individual votes. This also suggests that during the second round the nodes can send the blinded votes directly to $A_{q}$ without having to go around the ring, thus increasing the efficiency of the algorithm, as we will see in the experimental section. The new protocol is shown in Algorithm 2 . Round 2 is a degenerate one and can clearly be combined with Round 1.

The more efficient StR also provides an improvement over previous protocols in the field of secure multi-party sum com- 


$$
r_{l}=\sum_{j=1}^{n} r_{l, j}, \text { we obtain that } \quad r_{k}-r_{k, k}=\sum_{j \neq k} r_{k, j} \quad \text { and } \quad r_{l}-r_{l, l}=\sum_{j \neq l} r_{l, j} .
$$

Plugging these last two expressions to Equations (2) and putation [11]. In particular, in [11], a distributed potocol is

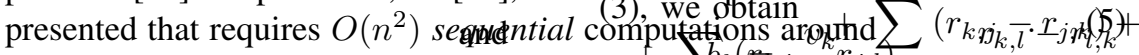

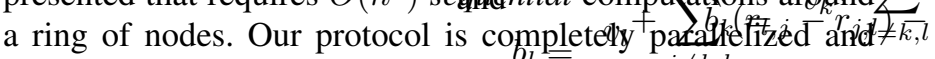

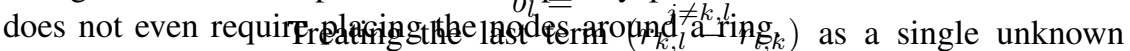
quantity, we see that it is impossible to correctly calculate the

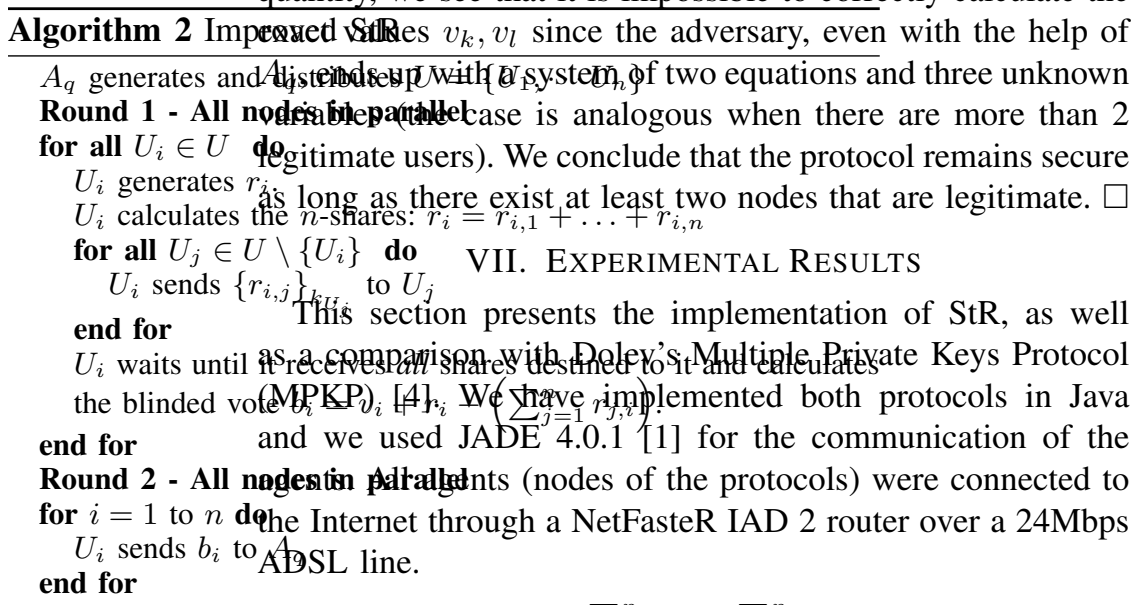
Upon reception of.alpyoteessitng çmputes $\sum_{i=1}^{n} b_{i}=\sum_{i=1}^{n} v_{i}$

The first phase of our experiments involved measuring the

In what followpegsing thesefustif. Doththemerefficiand decryption, we version of StR. used the RSA cryptosystem for encrypting the random shares

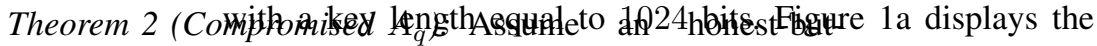

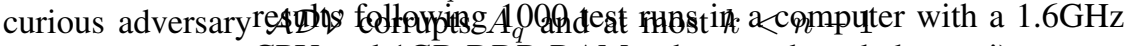

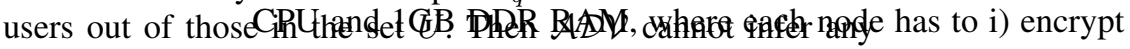

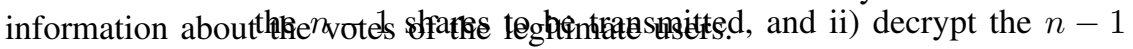
Proof. Here, we sbarcerequived treherease rankes from modes 5 to $n=100$.

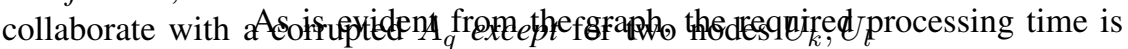
which are considefegligiblentande. does not constitute any real burden to nodes of

To prove that she Strotectsterel privacy of legitimate users,

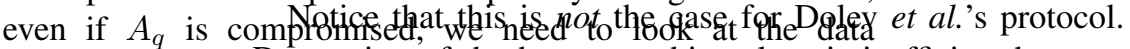
exchanged in StR. Recryptipn af the homemorphic, values is inefficient because

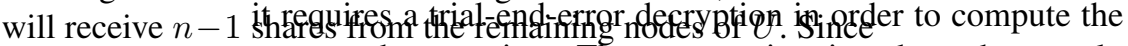
$n-2$ nodes are exprippontiseust ratipgs enthus, processing time depends not only

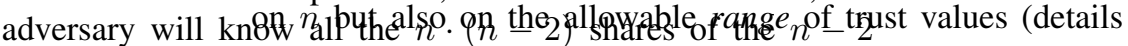
compromised nodemitted the $h$ to 4 space reftriqtions) Bespite this inefficiency, sent to the compromitreat oneth. times as comparable and we focus only on the

From the four remmmmications, aspects of both protocols only to $U_{k}$ and $U_{l B \text { sipes }}$. for the calculation of their blinded v vtes $b_{f}, h_{4}$. Additionally the last two remaining shares (r. to $U_{k}, U_{l}$ since they are encrypted phith their corresponding public keys and then exchanged between them. Since we have measure the communication dellay for the tirst round of StR. assumed that these two nodes are legitimate, they will not t. reveal the value of these shares to any other node.

To ease the analysis, in the following expressions we have

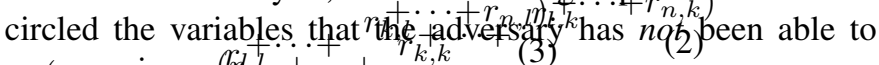
and $\quad r_{l}^{\text {cormpromise; } \frac{1}{k}}\left(r_{r_{1}, k}+\cdots+\right.$ $b_{l}=v_{l}^{+} \quad b_{k}=$ $v_{k}^{+}$ 


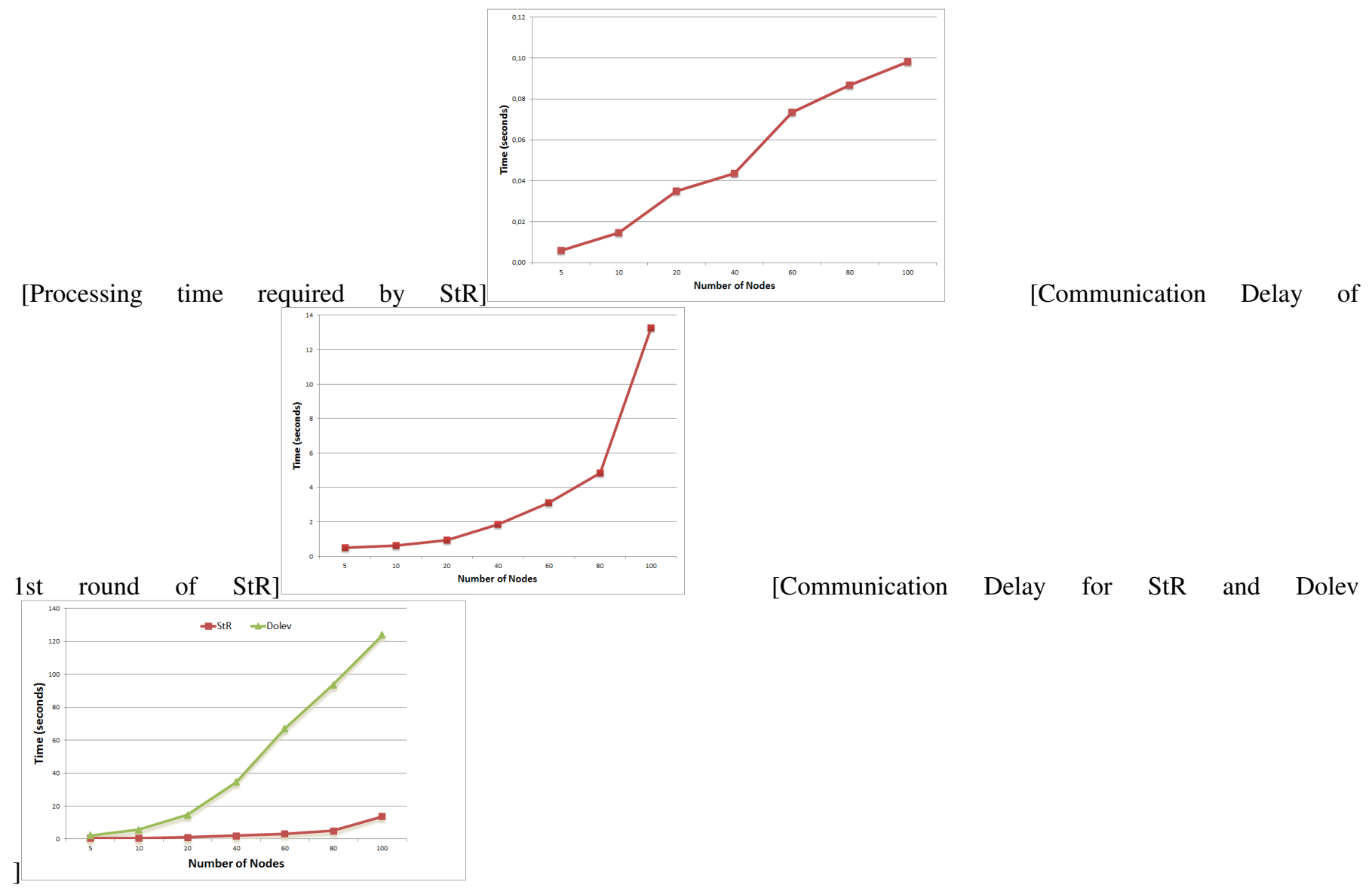

Fig. 1. Experimental Results — Processing Overhead \& Communication Delay

that generated $n$ encrypted shares (1024 bits long each); these were sent in parallel as single messages to each of the $n-1$ remaining nodes, where $n$ was incremented from $n=5$ to 100 in steps of 5 . As expected, the delay did not increase in a strictly linear manner, since the overhead processing of collecting the shares and computing the masked vote $b_{i}=v_{i}+r_{i}-\left(\sum_{j=1}^{n} r_{j, i}\right)$ increased with the number of nodes. Figure $1 \mathrm{~b}$ illustrates the delay in seconds as a function of the number of nodes $n$.

2) Second Round: While in StR only one message (the blinded vote) is transmitted from each node to $A_{q}$, this is not the case for Dolev's protocol as each node must send to the next one in the ring the result of the homomorphic encryption. Thus, in this case, we wanted to calculate the communication delay of transmitting a message of size 1024 bits long (the result of the homomorphic encryption) between successive nodes in the list $U$. We have run 1000 experiments in our JADE platform and we have found that, on average, the time to sent a single message between two successive nodes is approximately equal to 0.115 seconds.

We have summarized these findings in Figure 1c. This figure shows a comparison for the communication delay of both rounds of StR and Dolev's protocol. While both protocols show a quadratic behavior - Dolev's protocol sequentially propagates, for a total of $n$ times, a large message of length $O(n)$, while in StR each node sends, in parallel, $(n-1)$ messages of size $\mathrm{O}(1)$ - StR outperforms Dolev's protocol. This is something to be expected since during the first round of StR time is saved by sending the shares in parallel and not sequentially. Additionally, during the second round time is saved by eliminating the need to visit the nodes in the ring. Thus, the communication delay of StR for a list of up to one hundred voters, is almost an order of magnitude smaller than that of Dolev's protocol (13.7sec vs. 124sec) and is expected to be magnified even further for larger values of $n$.

\section{CONCLUSIONS}

In this work we presented StR, a decentralized privacyrespecting scheme for securely casting trust ratings in additive reputation systems. Our protocol has been formally proved to be resistant to collusion even against as many as $n-1$ malicious insiders. In the course of this work, we have also presented a lighter, but equally secure protocol, that can be thought as an independent contribution to the field of secure multiparty sum computation. The effectiveness of StR was demonstrated by conducting extensive experiments measuring its communication delay and processing overhead in a real $\mathrm{P} 2 \mathrm{P}$ network, showing its superior performance over the previous best protocol to date. 
As part of our future research, we intend to consider defense mechanisms that will effectively manage malicious adversaries, adversaries that deviate from the designated honestbut-curious behavior examined here.

\section{ACKNOWLEDGEMENTS}

This work has been funded by the European Community's FP7 project SafeCity (Grant Agreement no: 285556).

\section{REFERENCES}

[1] F. Bellifemine, A. Poggi, G. Rimassa, and T. Italia. Jade. Internal Tecnhical Report. http://jade.tilab.com/

[2] C. Clifton, M. Kantarcioglu, J. Vaidya, X. Lin and M. Y. Zhu. Tools for Privacy Preserving Distributed Data Mining. In ACM SIGKDD Explorations, 2003

[3] R. Dingledine, N. Mathewson, and P. Syverson. Reputation in P2P anonymity systems. Workshop on Economics of Peer-to-Peer Systems, 2003.

[4] S. Dolev, N. Gilboa, and M. Kopeetsky. Computing multi-party trust privately: in $o(n)$ time units sending one (possibly large) message at a time. ACM Symposium on Applied Computing (SAC '10), 2010.

[5] eBay. Buyer accountability. http://pages.ebay.com/services/forum/ sellerprotection.html.

[6] O. Hasan, L. Brunie, and E. Bertino. $k$-shares: A privacy preserving reputation protocol for decentralized environments. 25th IFIP International Information Security Conference (SEC 2010), 2010.

[7] A. Josang, R. Ismail, and C. Boyd. A survey of trust and reputation systems for online service provision. Decision Support Systems, 43:618644, Oct 2007.

[8] P. Paillier. Public-key cryptosystems based on composite degree residuosity classes. In Advances in Cryptology, EUROCRYPT '99, pp.223-238, 1999.

[9] E. Pavlov, J. S. Rosenschein, and Z. Topol. Supporting privacy in decentralized additive reputation. Second International Conference on Trust Management (iTrust 2004), pages 108-119, 2004.

[10] P. Resnick and R. Zeckhauser. Trust among strangers in internet transactions: Empirical analysis of ebay's reputation system. The Economics of the Internet and E-Commerce, 11(3):129-158, 2002.

[11] Rashid Sheikh, Beerendra Kumar, Durgesh Kumar Mishra. A Distributed $k$-Secure Sum Protocol for Secure Multi-Party Computations. In Journal of Computing, Volume 2, Issue 3, March 2010. 